\title{
Fuzzy Ant Colony Paradigm for Virtual Pedestrian Simulation
}

\author{
A. Boulmakoul* and M. Mandar
}

Faculté des Sciences et Techniques de Mohammedia (FSTM), Laboratoire Informatique de Mohammedia (LIM/IST), B.P. 146 Mohammedia, Maroc

\begin{abstract}
Pedestrian's studies are applied benefit and deployed in many areas; including public trip planning, and human factors analysis for building evacuation, or other situations where masses of people gather such as sporting events, etc. In this paper we describe a simulation system for virtual pedestrians. We mix the concept of fuzzy ant given by Ant Colony paradigm and associated cellular automaton model. We have adopted a fuzzy model for its particular ability to better represent uncertainty and imprecision about space perception. In our model we use it to represent virtual pedestrian's fuzzy pedestrian's desirability or visibility. A software solution was developed for this purpose. The Simulation results confirm predictions given by the first-order traffic flow theory. The proposed fuzzification is a preliminary step to consider other factors. The given system in its deployment phase will be integrated for pedestrians' accidents analysis in urban transportation networks. The strength of our model takes advantage in using explicit Ant Colony model and on projecting fuzzy theory to apprehend pedestrians' perceptions.
\end{abstract}

Keywords: Ant Colony Paradigm, cellular automata, intelligent agent, virtual pedestrian, fuzzy theory, simulation.

\section{INTRODUCTION}

Pedestrian's studies have attracted considerable researchers' attention. The reason for such study is that pedestrians movement and how it is affected by the design around, is an important component in design of transportation facilities, pedestrians walkways, building architectures, and other public buildings. Architects and designers have to take into consideration the characteristics of pedestrian flows in order to design the infrastructure as well as to assess its efficiency and safety [1]. In particular, a good understanding of the emergent patterns is required to predict how the flow will behave under different circumstances.

In real world, pedestrians move in two-dimensional spaces, and their complex behavior is easily affected by architectures surroundings. Scientific researchers focused on studying the characteristics of unidirectional and bidirectional counter pedestrian flows [2-6]. Their studies were conducted either by using models [2, 3, 7-11]; or by using empirical or experimental investigations with video analysis $[4,5,12-15]$.

Pedestrians flow models are grouped in three approaches: macroscopic, microscopic, and mesoscopic, which is the combination of two others. Generally pedestrians flow modeling can be performed by two first approaches. Macroscopic models treat the crowd as a whole, like a fluid or continuum [11, 16]. Microscopic models consider pedestrians as discrete particles in a computer simulation.

\footnotetext{
*Address correspondence to this author at the Faculté des Sciences et Techniques de Mohammedia (FSTM), Laboratoire Informatique de Mohammedia (LIM/IST), B.P. 146 Mohammedia, Maroc; Tel: 212233153 52; Fax: 212233153 53; E-mail: boulmakoul2000@yahoo.fr
}

Those models can be grouped into two categories: discrete, and continuous. Discrete models include cellular automaton $[3,10,17,18]$ and lattice gas $[13,17,19]$ models. Space and time are discretized to approximate the real movement of pedestrians. However, Most of those models, cannot compute precisely the distance and speed of pedestrian movement [17], and are ill suited for simulating two streams of populations obliquely intersecting because space is discretized into square lattices.

In continuous models, differential equation systems are used to describe the continuous movement of pedestrians in space and time. Those models introduce a concept of social force [8] and optimal control theory models [9].

Researchers discovered in nature a variety of interesting insects or animal behavior. A flock of birds' seeps across the sky, a group of ants' forages for food, a group of fish swims turns $[20,21]$. In spite of the simplicity of their individuals, present a highly structured social organization. This kind of aggregate motion is called "swarm behavior". To understand how these animals interact, achieve goals and evolve, biologists and computer scientists have studied how to model biology swarms. Furthermore, the engineers are more interested in this kind of swarm behavior, since the resulting "swarms intelligence" can be applied in optimization [22], in transportation systems engineering [23], in network development [24] and in robotics studies [20].

The $\mathrm{N}$ agents in the swarm cooperate to elaborate a part of a constant behavior and achieve certain purpose. This noticeable "collective intelligence" emerges from large groups of simple agents. The agents use simple local rules to perform their actions, and the swarm achieves its goals via the interaction of the entire group.

Swarm intelligence is the emergent collective intelligence of group of subsystem, which interacts relatively 
independently from all other subsystems. The autonomous subsystem doesn't follow commands from a leader or have some global plan [15]. For example, a bird in a flock has only to adjust its movement to coordinate with movements of its flock mates, particularly its "neighbors" which are close to it. The bird tries simply to stay close to its neighbors while avoiding collisions with them.

\section{MATERIALS AND METHODOLOGY}

In this work, we propose results of our research conducted in Mohammedia Computer Lab. These concern work for the PhD project of Meriem Mandar under the supervision of Professor Boulmakoul during the period 2008-2010.

This paper is structured as follows, after a short introduction, in section II, we recall some biological basis and foundation of artificial life; in section III, we introduce the concept of swarm intelligence. In section IV we present a state of the art about pedestrian simulation, followed by a new pedestrian fuzzy modeling. Section VI describes and completes our proposal model, and presents obtained results given by simulation.

\section{SWARM INTELLIGENCE}

Swarm intelligence is relatively new discipline, dealing with the study of self-organization processes, both in nature and artificial systems. Researchers in animal behavior have developed many models to explain interesting social insects' behavior, such as self-organization and shape formation. Algorithms and methods inspired by these models have been proposed to solve complex problems in many domains. An example of a particularly successful research direction in swarm intelligence is Ant Colony Optimization (ACO). It has been applied successfully to a large number of complex discrete optimization problems, including the travelling salesman problem (TSP), the quadratic assignment problem, and scheduling, vehicle routing, etc. Another interesting approach is focused on continuous optimization problems. Swarm robotics is another relevant field, where swarm intelligence techniques are applying to the control of large groups of cooperating autonomous robots.

Swarm intelligence describes a system of spatially distributed individuals coordinating their actions in a decentralized and self-organized way, to exhibit complex collective behavior. Such systems have a large number of individual's agents which interact with each other simply. This allows swarm intelligence systems to be robust and flexible. Moreover systems with those properties can range in size from nano to macro scale. Swarm intelligence systems are common throughout nature; samples are bacteria's colonies, and social insects. In addition human have developed a variety of artificial swarm systems ranging from swarm based optimization algorithms to sensor networks.

Since swarm intelligence involves the study of collective behavior in decentralized system, local interactions among the agents often cause global emergent patterns. Swarm intelligence models have many features in common with
Evolutionary Algorithms: they are population based; the system is initialized with a population of individuals, which are manipulated over many iteration steps. Unlike Evolutionary Algorithms, swarm intelligence models don't explicitly use evolutionary operators. An agent moves through the search space, by modifying itself according to its past experiences and to its relationship with other entities in the population and in the environment.

\section{PEDESTRIAN SIMULATION}

Both, inside public building and open areas, prediction of infrastructure influences planning on people behavior, comfort and mobility, become very important presses. Many studies have been carried out to evaluate these influences, either by traditional simulation tools or by developing modern pedestrian's simulation. Most of these models attempt to understand how space will affect crowd pedestrian flow, and how to improve space designing particularly in public transport. The ability of predicting pedestrian behavior is valuable in many urban planning contexts: architecture [26], marketing [27], and land use [28]. Especially, in panic situation [8-30] pedestrians behaviors' are dictated by a unique objective, saving their own life, consequently many of them become irrational [31].

Pedestrian simulation state of the art can be discussed around three types of models: (a) queuing models [32], in such models waiting times are introduced to deal with congestion phenomenon caused by traffic supply higher than infrastructure capacity. Works published by Christakos [32] using Helbing et al. simulation model [29] were used to precisely model these traffic problems. These models serve to describe pedestrian evacuation. (b) Cellular automata models divide space into similar cells, each cell has a set of rules, which defines its state at each time step.

Nagel and Schreckenberg introduced a model [33] to describe vehicles dynamics using a set of dynamics rules:

$$
\begin{aligned}
& \text { Acceleration } v_{i} \leftarrow \min \left(v_{i}+1, v_{\max }\right) \\
& \text { Bracking } v_{i} \leftarrow \min \left(v_{i}, g_{i}-1\right) \\
& \text { Randomization } v_{i} \leftarrow \max \left(v_{i}-1,0\right) \text { with probability } \mathrm{p} \\
& \text { Movement } x_{i} \leftarrow x_{i}+v_{i}
\end{aligned}
$$

Blue and Alderb model [2] define three fundamentals elements for pedestrian movement: (a) side stepping, (b) desired movement (acceleration or braking), (c) conflict mitigation. In [34] a conception of "stop point" is introduced to deal with traffic obstacles and resolve conflicts among pedestrians or between pedestrians and other vehicles on the crosswalk. Discrete choice models in general and random utility models [35], in particular are disaggregating behavioral models designed to forecast behavior of individuals in choice situations. In these models each alternative in a choice experiment can be associated with a value, called utility. The alternative with the highest utility is selected. The utility is modeled as a random variable depending on the attributes of alternatives and socioeconomic characteristics of the decision-maker. 
In his thesis, Mauron [36] has developed two approaches to simulate pedestrian behavior, the first one, uses the basic ideas given in [37]. This approach considers that the cells type is different (obstacle, link...); forces and walk directions are calculated at the start of simulation. The second one uses an environment with a set of point link. The agents use these links to find their path to destination. Continuous models are based on differential equations, the social force model [8] describes the pedestrian movement by the sum of acceleration, repulsive effect of others pedestrians, obstacles and attractive effects. Developed in context of artificial intelligence, agent based simulation of pedestrian behavior has been widely used in traffic simulation [38]. It provides a great deal of flexibility, as the behavior of each agent can be modeled independently, as a sequence of specific choices.

Complex dynamic and phenomena at an aggregate level of observation may emerge from interactions that occur at a local level. The multi-agent systems (MAS) offer the possibility to directly represent behaviors and interactions between individuals to explain and predict the evolution of complex phenomena. For instance, the simulation developed by Cavens et al. model [39] use multi-agents based simulation. Simulation software is divided into distinct modules, which interact with each other via network messages. The work presented by [40] presents a simulation of the hajj crowd based on the development on intelligent agent. Agents are able to recognize their environment and to adapt a rational behavior.

In real world, almost everything is relative and has a matter of degree. Human have a notable potential to accomplish a wide variety of mental and physical tasks without any prior measurements or computations. This ability is based on its perception. The probability theory, based on bivalent logic, shows an inability to operate on perception-based information. The bivalence of the conceptual structure of probability theory [41, 42] is in fundamental conflict with reality, in which almost everything has a degree of truth. It is this reality that is the point of departure in fuzzy theory.

\section{Fuzzy Numbers}

Non random impreciseness or vagueness occurs associated with numeric quantities in many human activities. This impreciseness may have different origins. For example, it is usual to say "he is about forty" or "I expect to make more than two million on this deal". The natural modeling tool for this kind of situation is the Theory of Fuzzy Sets. Given that precise numeric quantities are represented by real numbers, it should be thought that imprecise or vague quantities may be represented by "real fuzzy numbers".

Zadeh introduced the concept of an infinite valued logic [41] where he described fuzzy set theory mathematics and by extension fuzzy logic. The fuzzy concept can't be represented by the conventional approaches. Consequently the theory of the classic probability don't supply an abstract frame suited to represent the knowledge, because such a concept is lexically indistinct. Fuzzy set theory suggested making operate the values true and False on real numbers the interval $[0,1]$. New operations for the logic calculations were proposed as a generalization of the classic one. The work presented in [43] proposes the following definitions and perform a ranking method for fuzzy number.

Definition 1. (Fuzzy Set) Let $\mathrm{X}$ be a nonempty set. A fuzzy set $\mathrm{A}$ in $\mathrm{X}$ is characterized by its membership function $\mu_{A}: X \rightarrow[0,1]$.

$\mu_{A}(u)$ is interpreted as the degree of membership of element $\mathrm{x}$ in fuzzy set $\mathrm{A}$ for each $u \in X$. The set $\mathrm{A}$ is completely determined by the set of tuples $A=\left\{\left(u, \mu_{A}(u)\right) / u \in X\right\}$.

Definition 2. The membership function $f_{\tilde{A}}(x)$ of trapezoidal fuzzy number (TNF) $\tilde{A}(a, b, c, d)$ is defined by:

$$
f_{\tilde{A}}(x)= \begin{cases}f_{\tilde{A}}^{L}(x), & a \leq x \leq b, \\ \omega & b \leq x \leq c, \\ f_{\tilde{A}}^{R}(x), & c \leq x \leq d, \\ 0 & \text { otherwise }\end{cases}
$$

where $0 \leq \omega \leq 1$ is a constant $f_{\tilde{A}}^{R}:[c, d] \rightarrow[0, \omega]$ and $f_{\tilde{A}}^{L}:[a, b] \rightarrow[0, \omega]$ are two application strictly monotonous and continuous from $\mathrm{R}$ to a closed interval $[0, \omega]$. If $\omega=1$ then $\tilde{A}$ is called a normal fuzzy number. If the membership function $f_{\tilde{A}}(x)$ is linear piecewise then $\tilde{A}$ is called trapezoidal fuzzy number, denoted by $\tilde{A}(a, b, c, d ; \omega)$ or $\tilde{A}(a, b, c, d)$ if $\omega=1$.

Particularly, if $b=c$, the trapezoidal fuzzy number is reduced to a triangular fuzzy number denoted by $\tilde{A}(a, b, d ; \omega)$ or $\tilde{A}(a, b, d)$ if $\omega=1$.

Because of $f_{\tilde{A}}^{L}$ and $f_{\tilde{A}}^{R}$ are two application strictly monotonous continuous, then their reverse exist and must be also strictly monotonous continuous. Let $g_{\tilde{A}}^{L}:[0, \omega] \rightarrow[a, b]$ and $g_{\tilde{A}}^{R}:[0, \omega] \rightarrow[c, d]$ be the inverse application of $f_{\tilde{A}}^{L}$ and $f_{\tilde{A}}^{R}$ respectively. Then $g_{\tilde{A}}^{L}(y)$ and $g_{\tilde{A}}^{R}(y)$ must be integrals on closed interval $[0, \omega]$. In the case of trapezoidal fuzzy number, the reverse functions $g_{\tilde{A}}^{L}(y)$ and $g_{\tilde{A}}^{R}(y)$ may be analytically expressed by:

$$
\begin{array}{ll}
g_{\tilde{A}}^{L}(y)=a+(b-a) y / \omega, & 0 \leq y \leq \omega \\
g_{\tilde{A}}^{R}(y)=d-(d-c) y / \omega, & 0 \leq y \leq \omega
\end{array}
$$

In the following, Figs. $(\mathbf{1}, \mathbf{2})$ give respectively membership function and reciprocal member function of a trapezoidal fuzzy number. 


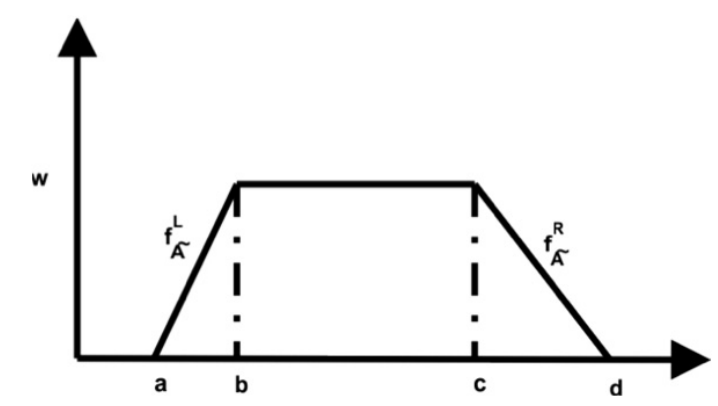

Fig. (1). Membership function of a trapezoidal fuzzy number.

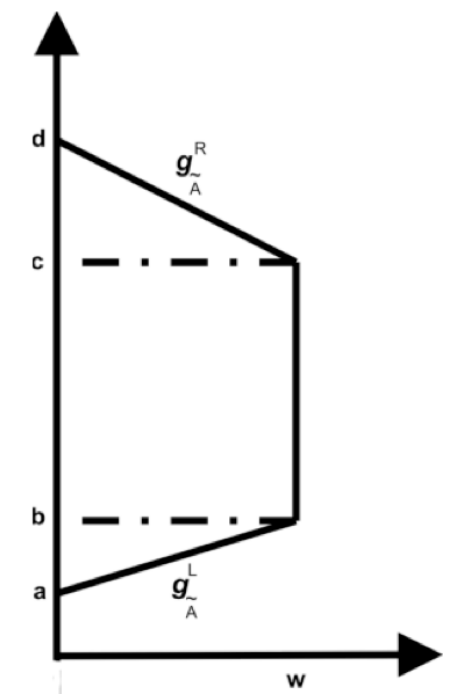

Fig. (2). Reciprocal member function of a trapezoidal fuzzy number.

The center point of a fuzzy number is given by:

$$
\begin{aligned}
& \overline{x_{0}}(\tilde{A})=\frac{\int_{-\infty}^{+\infty} x f_{\tilde{A}}(x) d x}{\int_{-\infty}^{+\infty} f_{\tilde{A}}(x) d x} \\
& =\frac{\int_{a}^{b} x f_{\tilde{A}}^{L}(x) d x+\int_{b}^{c}(x \omega) d x+\int_{c}^{d} x f_{\tilde{A}}^{R}(x) d x}{\int_{a}^{b} f_{\tilde{A}}^{L}(x) d x+\int_{b}^{c}(\omega) d x+\int_{c}^{d} f_{\tilde{A}}^{R}(x) d x} \\
& \overline{y_{0}}(\tilde{A})=\frac{\int_{0}^{\omega} y\left(g_{\tilde{A}}^{L}(y)-g_{\tilde{A}}^{R}(y)\right) d y}{\int_{0}^{\omega}\left(g_{\tilde{A}}^{L}(y)-g_{\tilde{A}}^{R}(y)\right) d y}
\end{aligned}
$$

So we can obtain

$$
\begin{aligned}
& \overline{x_{0}}(\tilde{A})=\frac{1}{3}\left[a+b+c+d-\frac{d c-a b}{(d+c)-(a+b)}\right] \\
& \overline{y_{0}}(\tilde{A})=\omega \frac{1}{3}\left[1+\frac{c-b}{(d+c)-(a+b)}\right]
\end{aligned}
$$

For a normal trapezoidal fuzzy number $\tilde{A}(a, b, c, d)$, we have $\overline{y_{0}}(\tilde{A})=\frac{1}{3}\left[1+\frac{c-b}{(d+c)-(a+b)}\right]$

Or for a triangular fuzzy number

$$
\begin{aligned}
& \overline{x_{0}}(\tilde{A})=\frac{1}{3}[a+b+d] \\
& \overline{y_{0}}(\tilde{A})=\omega \frac{1}{3}
\end{aligned}
$$

Particularly for a normal triangular fuzzy number $\overline{y_{0}}(\tilde{A})=\frac{1}{3}$

The ordering function of fuzzy number [45] is defines by $R(A)=\sqrt{\left(x_{0}\right)^{2}+\left(y_{0}\right)^{2}}$

So, let $\tilde{A}$ et $\tilde{B}$ two fuzzy numbers $\tilde{A} \leq \tilde{B} \Leftrightarrow R(A) \leq R(B)$

\section{Fuzzy Arithmetic}

Let $\tilde{A}\left(a_{A}, b_{A}, c_{A}, d_{A} ; \omega_{A}\right), \quad \tilde{B}\left(a_{B}, b_{B}, c_{B}, d_{B} ; \omega_{B}\right) \quad$ and $\tilde{C}\left(a_{C}, b_{C}, c_{C}, d_{C} ; \omega_{C}\right)$ be three fuzzy numbers, fuzzy operators can be expressed as follow:

$$
\begin{aligned}
& \tilde{A} \oplus \tilde{B}=\tilde{C}\left(a_{A}+a_{B}, b_{A}+b_{B}, c_{A}+c_{B}, d_{A}+d_{B} ; \min \left(\omega_{A}, \omega_{B}\right)\right) \\
& \tilde{A}-\tilde{B}=\tilde{C}\left(a_{A}-a_{B}, b_{A}-b_{B}, c_{A}-c_{B}, d_{A}-d_{B} ; \min \left(\omega_{A}, \omega_{B}\right)\right) \\
& \tilde{A} \otimes \tilde{B}=\tilde{C}\left(a_{A} * a_{B}, b_{A} * b_{B}, c_{A} * c_{B}, d_{A} * d_{B} ; \min \left(\omega_{A}, \omega_{B}\right)\right) \\
& \tilde{A} / \tilde{B}=\tilde{C}\left(a_{A} * d_{B}, b_{A} * c_{B}, c_{A} * b_{B}, d_{A} * a_{B} ; \min \left(\omega_{A}, \omega_{B}\right)\right)
\end{aligned}
$$

We recall some properties about operations of trapezoidal fuzzy number: Addition and subtraction between fuzzy numbers become trapezoidal fuzzy number; Multiplication, division, and inverse need not be trapezoidal fuzzy number Max and Min of fuzzy number is not always in the form of trapezoidal fuzzy number. But in many cases, the operation results from multiplication or division are approximated trapezoidal shape. For computational aspects, this is not limiting our approach

\section{PROPOSED MODEL}

\section{Cellular Automata}

Cellular automata is an artificial life approach to simulation modeling and is named after the principle of automata (entities) occupying cells according to localized neighborhood rules of occupancy. Each cell can have one of a finite number of states. Time and state variables are discrete which makes it ideally suited for efficient computer simulations of complex traffic phenomena. Cellular automata for pedestrian dynamics have been proposed for instance in $[3,18,21,44,45]$. These models can be considered as generalizations of the Biham-MiddletonLevine model for city traffic [46]. Almost all models have only nearest-neighbor interactions, except for the generalization proposed in [18] which is used for analyzing evacuation processes on-board passenger ships. The other 
models use a kind of "sub-lattice-dynamics" which distinguishes between different types of pedestrians according to their preferred walking direction. Such an update is not easy to generalize to more complex situations where the walking direction can change.

\section{Ant Colony Optimization}

Ant colony optimization is one of the most successful techniques in swarm intelligence. It is inspired by the pheromone trail laying and following behavior of ants. Such behaviors allow ant colonies and find shortest paths between their colonies and food sources. Ants communicate indirectly by the mine of chemical pheromone trials. In nature, ants usually walk randomly while laying down pheromone trials. If other ants find such a path, they don't keep walking randomly, but follow the trail and reinforcing it if they find food. However the pheromone evaporates with time passing. More ants will visit a shorter path and consequently the pheromone density remains high for a longer time. ACO algorithm requires defining the following [22, 47]:

- The problem needs to be represented appropriately, which would allow the ants to incrementally update the solutions through the use of a probabilistic transition rules, based on the amount of pheromone in the trail.

- A problem-dependent heuristic function $\eta$ that measures the quality of components that can be added to the current partial solution.

- A rule set for pheromone updating, which specifies how to modify the pheromone value $\tau$.

A probabilistic transition rule based on the value of the heuristic function $\eta$ and on the pheromone value $\tau$ is used to iteratively construct a solution.

Starting from its start node, an ant iteratively moves from one node to another. When being at a node, an ant chooses to go to an unvisited node at time $t$ with a probability given by

$$
P_{i j}^{k}(t)=\left\{\begin{array}{lr}
\frac{\left[\tau_{i j}(t)\right]^{\alpha}\left[\eta_{i j}(t)\right]^{\beta}}{\sum_{l \in J_{l}^{T_{k}}}\left[\tau_{i l}(t)\right]^{\alpha}\left[\eta_{i l}(t)\right]^{\beta}} & \text { If } \quad j \in J_{i}^{T_{k}} \\
0 & \text { otherwise }
\end{array}\right.
$$

where $N_{i}^{k}$ is the neighborhood of the ant k, that is; $\tau_{i j}(t)$ is the pheromone value on the edge (i,j) at the time t, $\alpha$ is the weight of pheromone; $\eta_{i j}(t)$ is a priori available heuristic information on the edge $(i, j)$ at the time $t, \beta$ is the weight of heuristic information. Parameters $\alpha$ and $\beta$, determine the relative influence of pheromone trail. Heuristic information. $\tau_{i, j}(t)$ is determined by:

$$
\tau_{i j}(t)=\rho \tau_{i j}(t-1)+\sum_{k=1}^{m} \tau_{i j}^{k}(t) \quad \forall(i, j)
$$

$\Delta \tau_{\mathrm{ij}}{ }^{\mathrm{k}}=\mathrm{Q} / \mathrm{L}_{\mathrm{k}}(\mathrm{t})$ iIf the edge is $(\mathrm{i}, \mathrm{j})$ is choosen by the ant $\mathrm{k}_{\mathrm{k}}$ and is equal to zero otherwise where $\rho$ is the pheromone trail evaporation rate $0<\rho<1, \mathrm{~m}$ is the number of ants, $\mathrm{Q}$ is a constant for pheromone updating.

A generalized version of the pseudo-code for the ACO algorithm with reference to the TSP is illustrated in the following Algorithm.

\section{Ant Colony Optimization Algorithm}

1. Initialize the number $m$ of ants, and other parameters.

2. While (the end criterion is not met) do

3. $t=t+1$;

4. For $k=1$ to $n$

5. $\quad a_{k} t_{k}$ is positioned on a starting node;

6. For $m=2$ to problem_size

7. Choose the state to move into

8. According to the probabilistic transition rules;

9. Append the chosen move into $\operatorname{tabu}_{k}(t)$ ant $_{k}$;

10. Next $m$

11. Compute the length $L_{k}(t)$ of the tour $T_{k}(t)$ chosen by the ant ${ }_{k}$;

12. Compute $\Delta \tau_{i j}(t)$ for every edge $(i, j)$ in $T_{k}(t)$ according to Equation (1);

13. Next $k$

14. Update the trail pheromone intensity for every edge $(i, j)$ according to Equation (2)

15. Compare and update the best solution;

16. End While.

\section{Model Rules}

Each pedestrian has a preferred movement direction, a preferences matrix $3 \times 3$ contains movement preferences for 8 neighbors cells (Fig. 3), central element define pedestrian position, at each time step, a new fuzzy preference matrix is assigned to each pedestrian. Preference matrix is firstly proposed in [3].

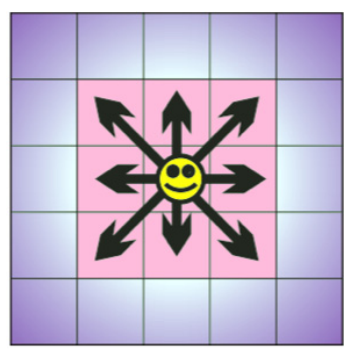

Fig. (3). Moore neighbor for Pedestrian with possible transitions.

For every pedestrian in cell (i):

1. A fuzzy matrix $\tilde{P}_{i j}$ of preference is assigned, which reflect movement desirability to one of eight neighbors cells

2. A fuzzy static influence $\tilde{S}_{j}$ of fix object on grid (obstacles), while $\mathrm{j}$ is the destination cell;

3. The movement possibility toward a cell depend o its 
occupation state $\left(O_{j}=1\right.$ or $\left.O_{j}=0\right)$. We have chosen to not represent this parameter as a fuzzy number because it doesn't require a degree of correctness. Each cell can hold just one person per time, and its size is set to fit this matter.

4. A fuzzy general utility of movement from a cell (i) to a cell $(\mathrm{j})$ is given by:

$\tilde{U}_{i j}^{k}(t)= \begin{cases}\frac{\left[\tilde{\tau}_{i j}(t)\right]^{\alpha}\left[\tilde{\eta}_{i j}(t)\right]^{\beta}}{\sum_{l \in J_{l}^{T_{k}}}\left[\tilde{\tau}_{i l}(t)\right]^{\alpha}\left[\tilde{\eta}_{i l}(t)\right]^{\beta}} & \text { If } \quad j \in J_{i}^{T_{k}} \\ 0 & \text { otherwise }\end{cases}$

while $\tilde{\tau}_{i j}:$ pheromone quantity ;

$\alpha$ : influence control parameter of $\tilde{\tau}_{i j}$

The pedestrian desirability or visibility is given by: $\tilde{\eta}_{i j}=\tilde{P}_{i j} \times \tilde{S}_{j} \times\left(1-O_{j}\right)$

where $\beta$ : influence control parameter of $\tilde{\eta}_{i j}$

$J^{T_{k}}$ : set of eight cells neighboring cell (i).

5. Pheromone update rule is given by:

$\tau_{i j}=\rho \tau_{i j}+\Delta \tau_{j}$

where $\rho$ is pheromone vaporization rate.

$\Delta \tau_{j}=\sum_{k=1}^{m} \Delta \tau_{j}^{k}(t)$ is the sum of pheromone layed down by all pedestrians at time step t. in a time step only one pedestrian occupy a cell, so $\Delta \tau_{j}^{k}(t)=O_{j}(t)$

6. Pedestrians move to cell (j) where the fuzzy general utility obtains its maximum value : $\tilde{U}_{i j}^{k}(t)=\max _{k}\left(\tilde{U}_{i k}^{k}(t)\right)$

\section{Conflicts Mitigation}

Pedestrian collision means two pedestrian will move into a same cell in time step $(t+1)$ (Fig. 4). A solution is proposed to avoid this problem is based on general utility toward destination cell. Pedestrian having higher general utility is able to execute his step.

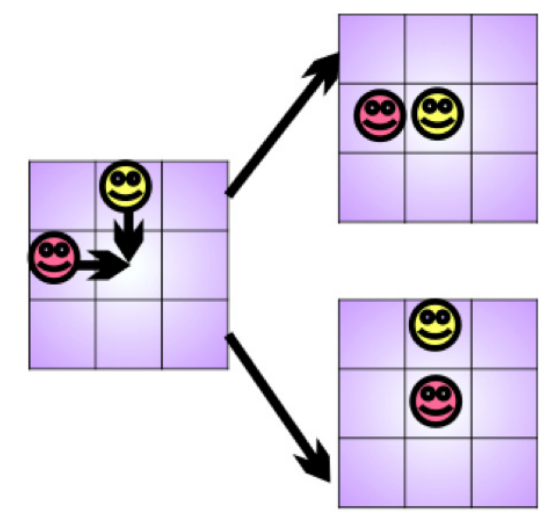

Fig. (4). Proposed solution for a conflict situation.
$\tilde{R}_{1}=$ fuzzy general utility value for pedestrian 1 for shared destination cell.

$\tilde{R}_{2}=$ fuzzy general utility value for pedestrian 2 for shared destination cell.

If $\tilde{R}_{1}>\tilde{R}_{2}$ then it's pedestrian who will move, else it's pedestrian 2 .

In the following figure (Fig. 5) we represent our model algorithm.

In this model, the fuzzification of pedestrians' utility concerns only spatial perception (obstacles, preferred direction, amount of pheromone for dynamic floor, etc.). Our goal in this first approach is to have a simple model integrating fuzzy modeling and the Ant Colony paradigm. Certainly, other cognitive and behavioral factors will be considered in our future work. This work is scheduled to consider dangerousness of crossing intersections by pedestrians. Perception of vehicle speed by pedestrians and other psychological factors can be integrated. The software architecture of the simulator allows this extension. For theoretical foundation, the fuzzy general utility proposed here, may be interpreted as a fuzzy probability, extending the crisp probability transition given by Ant Colony paradigm.

\section{Comparison of Pedestrian Simulation Models}

Generally in microscopic pedestrian simulation models, the pedestrian's movement is directed by the use of two terms. The first express the goals attractivity, and the other is used for expressing a repulsive effect to avoid collision with obstacles and others pedestrians. To classify the different modeling approach, several way of classification could be given: macroscopic versus microscopic description, rules based versus force-based interactions, stochastic versus deterministic dynamics, and discrete versus continuous variables. We adopt this last criterion to classify some major microscopic pedestrian's simulation models in comparison with ours (see Table 1).

The fuzzy modeling method presented in this paper exploits explicitly the Ant Colony paradigm (ACP). Our contribution is offered for the first time in this work. First we confirm the validity of the model to capture physical phenomenon of traffic theory and secondly it give first attempt to ACP fuzzification for modeling pedestrians' behavior. Obviously other factors or cognitive perceptions variables will be considered for model extension. We have used preference matrix as in work given in [3], to allow partial integration of the motivations of pedestrians. This preference will be modeled by a scheme of rules based on other cognitive and behavioral factors.

\section{RESULTS AND DISCUSSION}

Physical Characteristics

Pedestrian flow: The flow is usually defined as the number $\mathrm{N}(\mathrm{x}, \mathrm{t})$ of pedestrians passing a measurement point $\mathrm{x}$ between times $\mathrm{t}$ and $t+\Delta t$ 


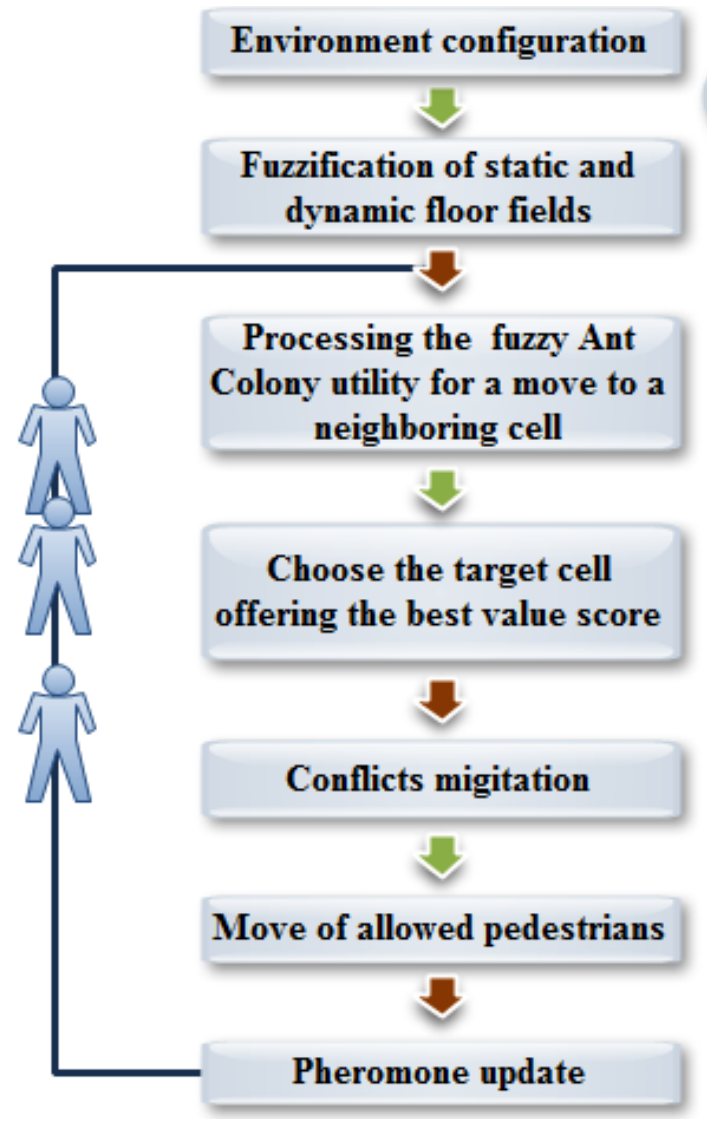

\section{set static objects: obstacles and pedestrian generators}

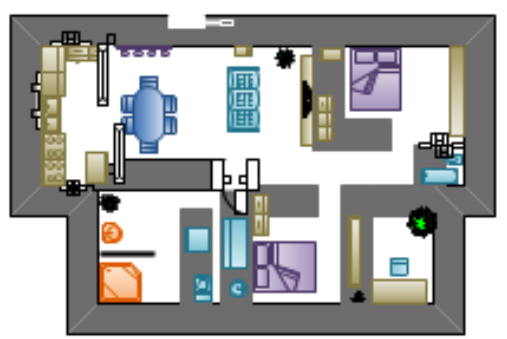

Fig. (5). Simulation's algorithm.

$\varphi(x, t, t+\Delta t)=\frac{N(x, t, t+\Delta t)}{\Delta t}$

The supply is a term that is connected to the flow, it represents the flow of pedestrians, in the absence of congestion, arriving to a section of a path and wanting to be served. In the ideal case of traffic, the flow is maximum and it is equal to the capacity of the used path. The pedestrian flow on a fixed site $i$, is given by:

$\Phi=\frac{1}{T} \sum_{t=t}^{t} \sum_{0}^{+T} n_{i, i+1}(t)$

where $n_{i, i+1}(t)=1$ if and only if a motion of a pedestrian was detected between sites $i$ and $i+1$ ( 0 otherwise).

Pedestrian density: The density is the number of pedestrians existing between $\mathrm{x}$ and $x+\Delta x$ at time $\mathrm{t}$

$\rho(t, x, x+\Delta t)=\frac{N(t, x, x+\Delta t)}{\Delta x}$

Since virtual pedestrians moves through a lattice's sites, their density in a given one $\mathrm{i}$ (= occupation of this site), is the sum of its occupations during a period of time T. The pedestrian density is given by: $\rho=\frac{1}{T} \sum_{t=t_{0}+1}^{t_{0}+T} n_{i}(t)$ where $n_{i}(t)=0$ if and only if the site is empty ( 1 if occupied) per unit time. $\mathrm{T}$ defines the period of time, and $n_{i}$ the occupation of the site $i$.

Fundamental diagram: Several experimental studies of the flow of traffic have confirmed the existence of a significant effect, which is the reduction of average speed with increasing pedestrian density. The first relationship of the fundamental diagram $q(\rho)$ was based on this effect [49]. The relationship describing the fundamental diagram can be obtained from the relationship between the average velocity $v(\rho)$ and density $\rho$.

For very low density, average speed tends to the maximum value $v_{\max }$, and for a maximum density, pedestrian movement becomes impossible. So we can write

$$
v\left(\rho_{0}\right)=v_{\max } \text { and } v\left(\rho_{\max }\right)=0
$$

The fundamental equation of flow, from which we can trace the fundamental diagram flow-density relationship is given by the hydrodynamics equation, which links flow q to velocity v:

$$
\rho(t)=\frac{q(t)}{v(t)}
$$


Table 1. Microscopic Pedestrians Models Comparison

\begin{tabular}{|c|c|c|c|c|c|}
\hline & \multicolumn{3}{|c|}{ Discrete Models } & \multicolumn{2}{|c|}{ Continuous Models } \\
\hline & CA Models $[2,3,34,35,37]$ & $\begin{array}{l}\text { Benefit Cost } \\
\text { Cellular }[48]\end{array}$ & $\begin{array}{l}\text { Fuzzy Ant Model } \\
\text { (Boulmakoul } \text { et al.) }\end{array}$ & Magnetic Force [33] & $\begin{array}{c}\text { Social Force } \\
{[8,29]}\end{array}$ \\
\hline Space and time & Discrete & Discrete & Discrete & Continuous & Continuous \\
\hline Variables State & Discreet & Discrete & Discrete & Continuous & Continuous \\
\hline Movement to goal & An objective function & Gain score & Fuzzy utility & $\begin{array}{l}\text { Positive and negative } \\
\text { magnetic force }\end{array}$ & Intended velocity \\
\hline Repulsive effect & $\begin{array}{l}\text { Conflict mitigation between } \\
\text { pedestrians, plus cells state for } \\
\text { obstacles }\end{array}$ & Cost Score & Static floor field & $\begin{array}{c}\text { Repulsive force plus } \\
\text { force to avoid collision } \\
\text { with other pedestrian or } \\
\text { obstacles }\end{array}$ & Interaction forces \\
\hline Attractive effect & Cell state for obstacles & Score value & $\begin{array}{l}\text { Fuzzy dynamic floor } \\
\text { field }\end{array}$ & $\begin{array}{l}\text { Attractive force } \\
\text { between goals }\end{array}$ & $\begin{array}{l}\text { Interaction } \\
\text { Forces }\end{array}$ \\
\hline Pedestrian movement & Discrete & Discrete & Discrete & Continuous & Continuous \\
\hline Value of variables & arbitrary & arbitrary & Bio-inspired meaning & Physical meaning & $\begin{array}{l}\text { Physical } \\
\text { meaning }\end{array}$ \\
\hline Interaction & Rules based & Rules based & Rules based & Force based & Force based \\
\hline Phenomena explained & $\begin{array}{l}\text { Queuing, } \\
\text { Way finding, } \\
\text { Shortest path } \\
\text { Self-organization } \\
\text { evacuation }\end{array}$ & queuing & $\begin{array}{l}\text { Queuing, } \\
\text { Way finding, } \\
\text { Shortest path } \\
\text { Self-organization } \\
\text { evacuation }\end{array}$ & $\begin{array}{c}\text { Queuing, } \\
\text { Way finding, } \\
\text { evacuation }\end{array}$ & $\begin{array}{l}\text { Queuing, } \\
\text { Self- } \\
\text { organization, } \\
\text { Oscillatory } \\
\text { change }\end{array}$ \\
\hline $\begin{array}{c}\text { Higher programming } \\
\text { orientation }\end{array}$ & Cellular based & Cellular based & $\begin{array}{l}\text { Bio-inspired } \\
\text { Cellular based } \\
\text { Fuzzy Logic }\end{array}$ & Heuristic & mathematics \\
\hline $\begin{array}{l}\text { Evacuation } \\
\text { application }\end{array}$ & possible & possible & possible & possible & Not possible \\
\hline $\begin{array}{l}\text { Parameters } \\
\text { calibration }\end{array}$ & By inspection & By inspection & By inspection & By inspection & By inspection \\
\hline
\end{tabular}

From this function we can trace the fundamental diagram $q(\rho)$. The flow becomes null if the density is null or maximum, and takes a maximum value at intermediate densities. It is often assumed that the fundamental diagram is composed of two isolated curves, which are separated by a threshold: a curve with a positive slope at low densities (it is the regime of free flow of traffic) and a curve with a negative slope to high densities (it is the regime of congested traffic flow) [50-52].

We have chosen a triangular fuzzy number representation to represent model parameters. For the simulation parameters and for a fixed value of static and dynamic influence, we have changed in each run the alpha parameter value of a fuzzy number for the preferences matrix element. This allows us to vary preference matrix from a crisp number to a fuzzy interval. We also change the influence parameter for static and dynamic floor. Some curves illustrating the characteristics of pedestrian traffic are given in the following. Chosen Simulation scenario (Fig. 6) consists on a room with two entries and one exit, witch contains many obstacles.

\section{Simulation Results for Pedestrian Traffic Parameters}

Influence Parameters of floor field $\alpha=1 \beta=1$

During simulation (Fig. 6), we noticed at room's exit, that pedestrian's density increases until it reaches a maximum value when traffic becomes congested (see Fig. 7). While pedestrians flow decreases in the same phase (see Fig. 8). The fundamental diagram (see Fig. 9) illustrates the phases of the free and congested pedestrian's traffic. Average evacuation time for pedestrians increases while the width of preference matrix increases (Fig. 10). Pedestrians move according to their preference matrix, and the fact of increasing the width of its components implies that the fuzzification threshold also increases. This does not allow pedestrians to find their way out quickly. As for a macroscopic traffic model, these results show that with fuzzyfication of the ant basic model, we can obtain results physically significant, such as the fundamental diagram which considers two phases: free flow and congestion (see Figs. 7-10). 


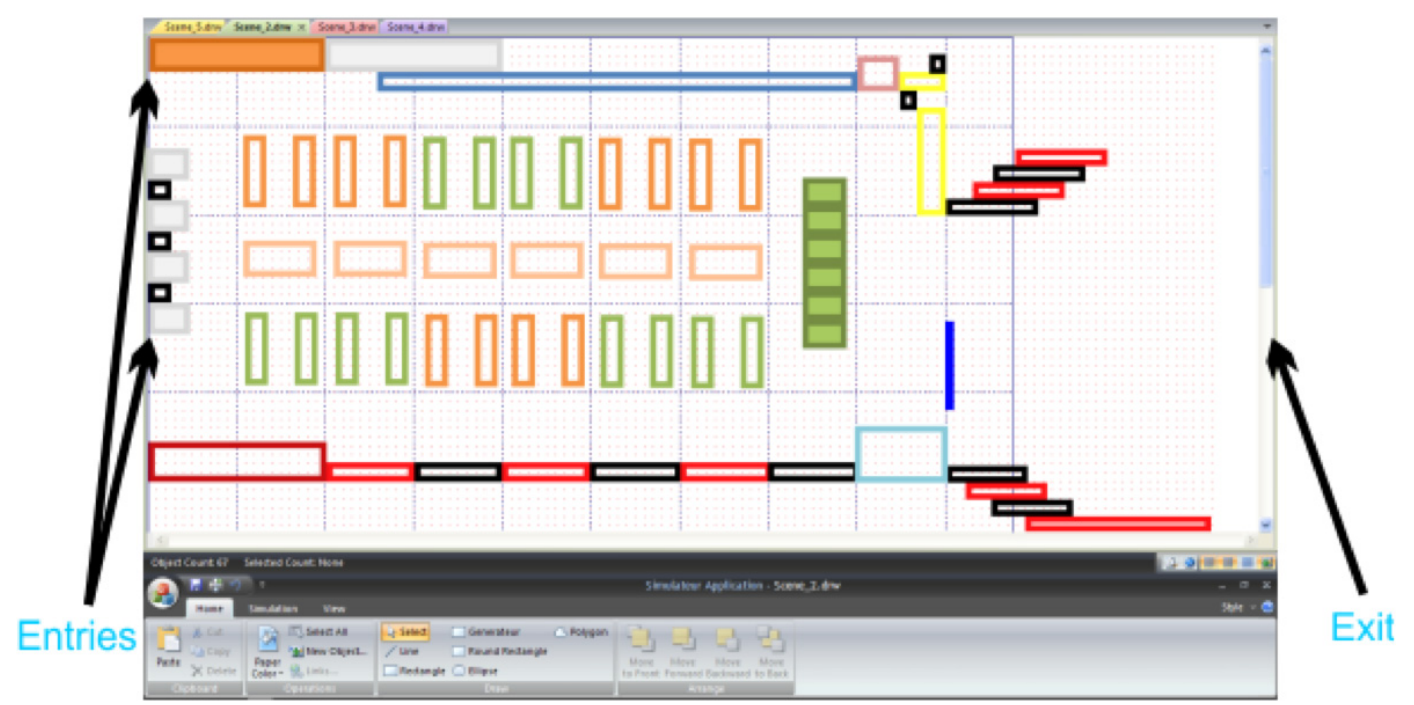

Fig. (6). Simulation scenario with two entries and an exit.

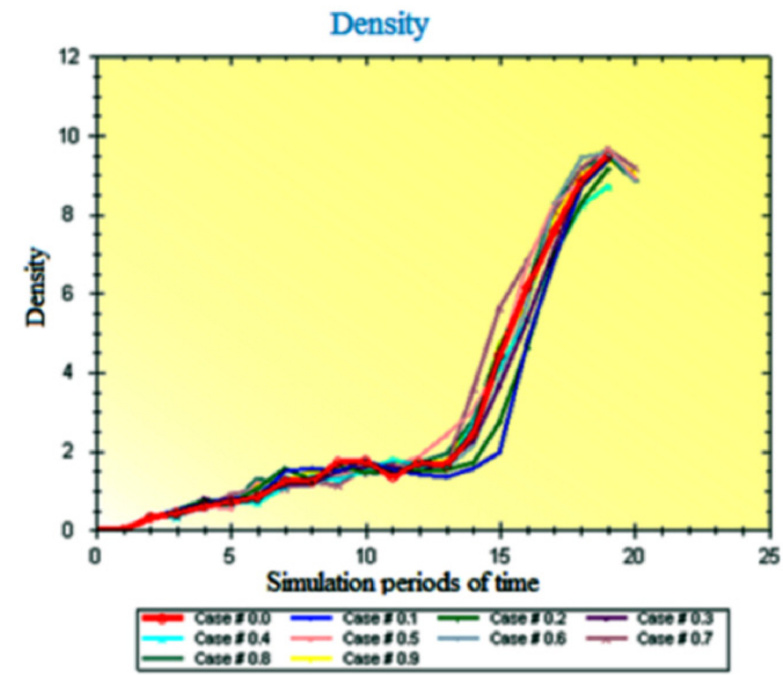

Fig. (7). Pedestrian density diagram.

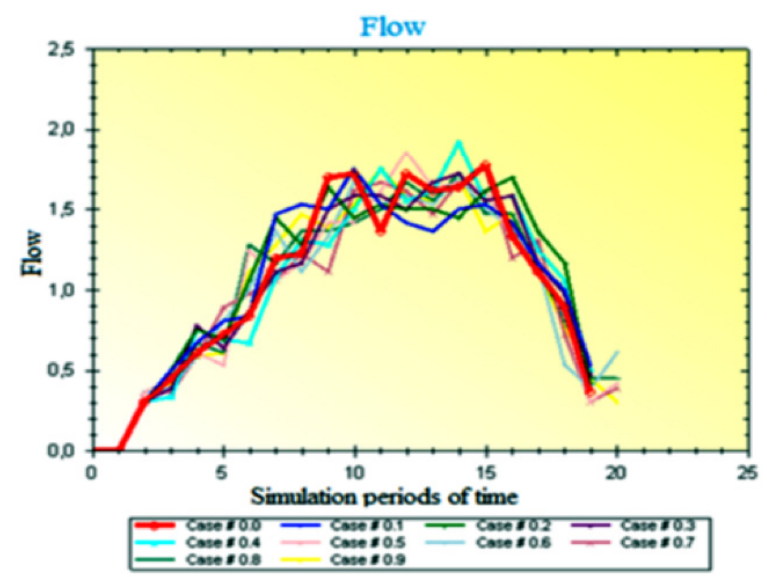

Fig. (8). Pedestrian flow diagram.

While choosing a simulation scenario as closed corridor (Fig. 11), pedestrians move forward with the same speed until they are blocked either by the corridor or by other blocked pedestrians. They form therefore a spreading wave of chock as shown in Fig. (12).

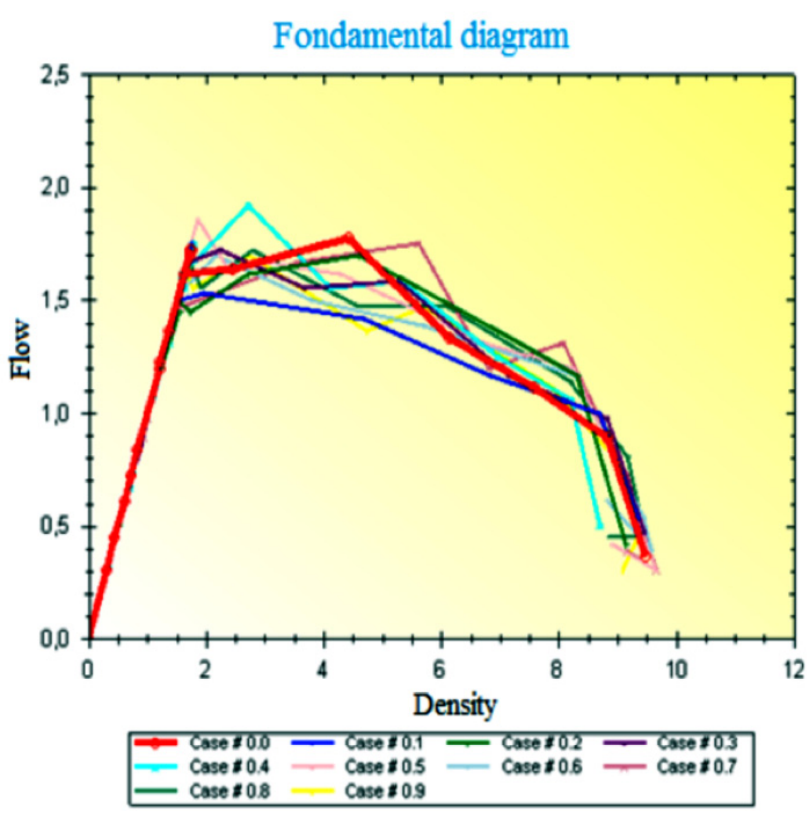

Fig. (9). Pedestrian traffic fundamental diagram.

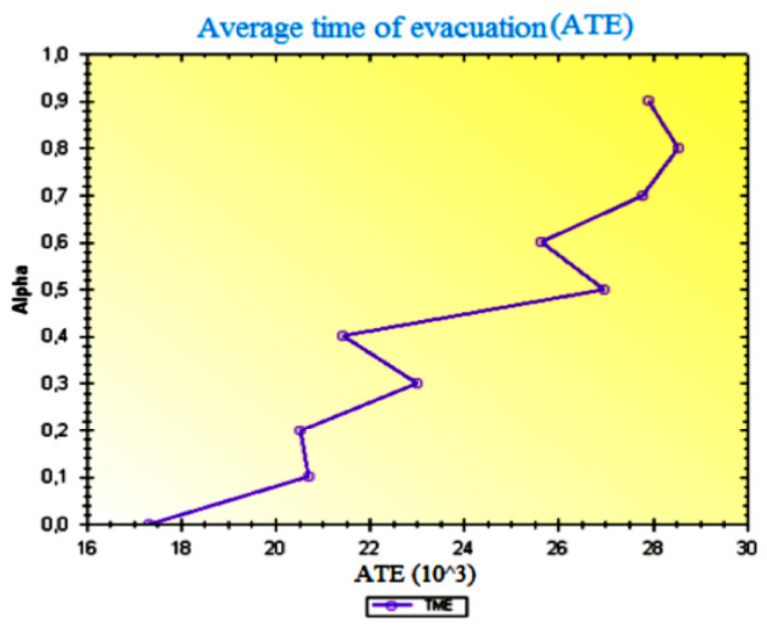

Fig. (10). Average evacuation time depending on the width of preference matrix. 
In Fig. (13) an open corridor with two pedestrians' generators with opposite motion direction, has been simulated. We notice that after some simulation time steps, pedestrians have been able to find their way by forming lanes with same direction.

\section{Scenario of Blocked Corridor}

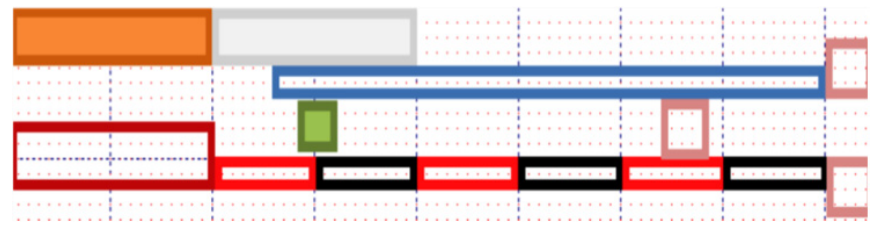

Fig. (11). Simulation scenario as a blocked corridor.

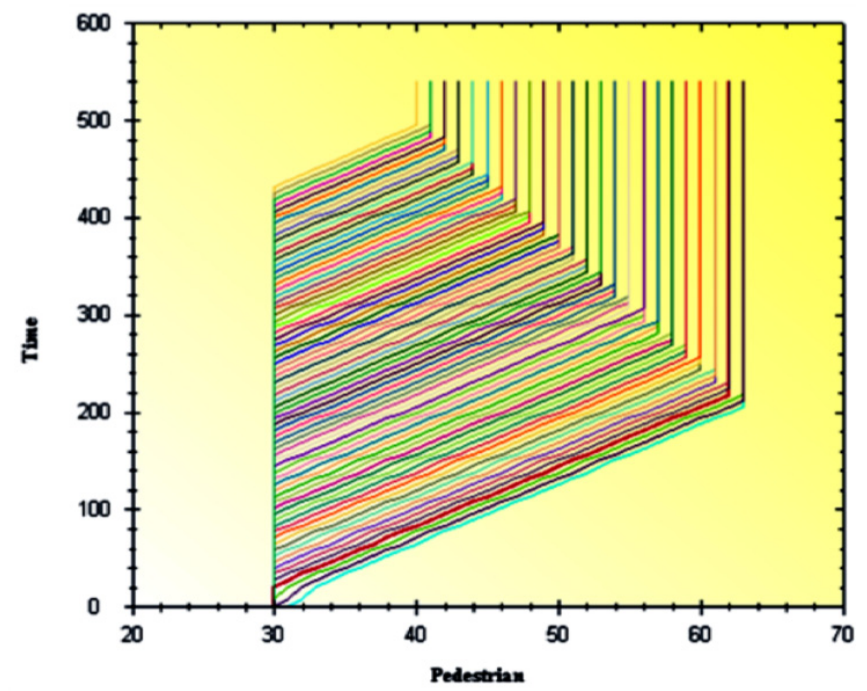

Fig. (12). Dissemination of a shock wave of pedestrians moving in time according to their positions.

\section{Scenario of Open Corridor}

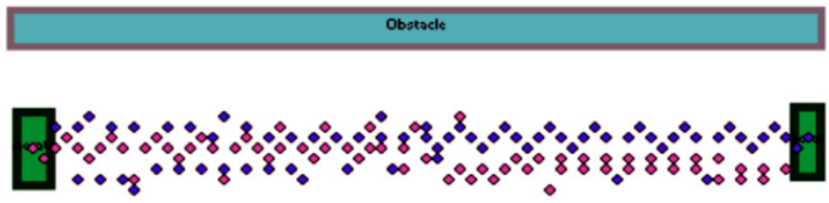

\section{Obstocle}

Fig. (13). Two crossing pedestrians lanes, with opposite direction.

\section{CONCLUSION}

In this paper, we present a simple fuzzy virtual pedestrian simulation model. Our model uses the basic ant model given by Ant Colony paradigm, to which we have integrated a fuzzy description to better represent, preferences matrix and chemical trace. Relation between density and velocity of pedestrian movement has so far mainly been analyzed using an empirical approach and fundamental relations found from the fitting of experimental measurements of the main quantities. Simulation results confirm predictions given by the fir-order traffic flow theory. Validation of the simulation model toward the real world data is recommended for further study. In our future work we plan to study the interactions between pedestrians and vehicles, to estimate the risk of crossing intersections. In our future work, fuzzification for other perception's factors of virtual pedestrian will be considered, such as: distance to an obstacle, crossing the road speed, etc.

\section{REFERENCES}

[1]
Dijkstra J, Timmermans HJP, Jessurun AJ. A multi-agent cellular automata system for visualising simulated pedestrian activity. In: Proceedings on the $4^{\text {th }}$ International Conference on Cellular Automata for Research and Industry: Theoretical and Practical Issues on Cellular Automata. Oct. 4-6, 2000, pp. 29-36, Germany. Blue VJ, Adler JL. Cellular Automata Model of Emergent Collective Bi-Directional Pedestrian Dynamics. In Artificial Life Bedau MA VII, McCaskill JS, Packard NH, Rasmussen S, Eds. MIT Press 2000. Available from: http://www.ulster.net/ vjblue/re search.htm

Burstedde C, Klauck K, Schadschneider A, Zittartz K. Simulation of pedestrian dynamics using a two-dimensional cellular automaton. Phys Stat Mech Appl 2001; 295: 507-25.

Kretz T, Grunebohm A, Kaufman M, Mazur F, Schreckenberg M. Experimental study of pedestrian counter flow in a corridor. J Stat Mech 2006; 56: P10001.

Lam WHK, Lee JYS, Chan KS, Goh PK. A generalized function for modeling bi-directional flow effects on indoor walkways in Hong Kong. Transp Res 2003; 37: 789-810.

Lam WHK, Lee JYS, Cheung CY. A study of the bi-directional pedestrian flow characteristics at Hong Kong signalized crosswalk facilities. Transportation 2002; 29: 169-92.

Antonini G, Bierlaire M, Weber M. Discrete choice models of pedestrian walking behavior. Transp Res Part B: Methodol 2006; 40: 667-87.

Helbing D, Farkas I, Vicsek T. Simulating dynamical features of escape panic. Nature 2007; 407: 487-90.

Hoogendoorn SP, Bovy P. Pedestrian route-choice and activity scheduling theory and models. Transp Res Part B 2004; 38: 169-90. Huang L, Xia Y, Wong SC, Shu CW, Zhang M, Lam WHK. Revisiting hughes dynamic continuum model for pedestrian flow and the development of an efficient solution algorithm. Transp Res Part B: Methodol 2009; 43: 127-41.

Hughes RL. A continuum theory for the flow of pedestrians. Transport Res Part B: Methodol 2002; 36: 507-35.

Daamen W, Hoogendoorn SP. Experimental research of pedestrian walking behaviour. Transp Res Rec 2003; 1828: 20-30.

Helbing D, Isobe $M$, Nagatani $T$, Takimoto $K$. Lattice gas simulation of experimentally studied evacuation dynamics. Phys Rev E Stat 2003; 67: 067101.

Helbing D, Buzna L, Johansson A, Werner. Self-organized pedestrian crowd dynamics: Experiments, simulations, and design solutions. Transp Sci 2005; 39: 1-24.

Hoogendoorn SP, Daamen W. Pedestrian behavior at bottleneck. Transport Sci 2005; 39: 147-59.

Huang L, Xia Y, Wong SC, Shu CW, Zhang M, Lam WHK. A dynamic continuum model for bi-directional pedestrian flows. Comput Mech 2009; 162: 67-75.

Guo RY, Huang HJ. A mobile lattice gas model for simulating pedestrian evacuation. Phys A: Stat Mech Appl 2008; 387: 580-6.

Kirchner A, Schadschneider A. Simulation of evacuation processes using a bionics-inspired cellular automaton model for pedestrian dynamics. Phys A: Stat Mech Appl 2002; 312: 260-76.

Fukamachi M, Nagatani T. Sidle effect on pedestrian counter flow. Phys A 2007; 377: 269-78.

Venayagamoorthy GK, Grant LL, Doctor S. Collective robotic search using hybrid techniques: Fuzzy logic and swarm intelligence inspired by nature. Eng Appl Artif Intellect 2009; 22: 431-41.

Wen-San H. Foraging behaviors of two sympatric ant species in response to lizard eggs. Zoology 2010; 113(2): 85-90.

Bonabeau E, Dorigo M, Theraulaz G. Swarm intelligence: from natural to artificial systems. New York, NY: Oxford University Press 1999.

3] Teodorović D. Swarm intelligence systems for transportation engineering: Principles and applications. Transp Res Part C: Emerg Technol 2008; 16: 651-67.

Saleem M, Di Caro GA, Farooq M. Swarm intelligence based routing protocol for wireless sensor networks: Survey and future directions. Inform Sci 2010; doi: 10.1016/j.ins.2010.07.005. 
[25] Flake GW. The computational beauty of nature: computer explorations of fractals, chaos, complex systems and adaptation. Cambridge, MA: MIT Press 1999.

[26] Okazaki S. A study of pedestrian movement in architectural space. Part 1: pedestrian movement by the application of magnetic models. Trans AIJ 1979; 283: 111-9.

[27] Borgers A, Timmermans H. A model of pedestrian route choice and demand for retail facilities within inner-city shopping areas, Geogr Anal 1986; 18 (2): 115-28.

[28] Pachter M, Chandler PR. Challenges of autonomous control: IEEE Cont Syst Mag 1998; 18: 92-7.

[29] Helbing D, Farkas I, Molnar P, Vicsek T. Simulation of pedestrian crowds in normal and evacuation simulations. In: Schreckenberg M, Sharma S, Eds. Pedestrian and Evacuation Dynamics, Duisburg 2002; pp. 21-58.

[30] Klupfel H, Meyer-Konig T, Wahle J, Schreckenberg M. Microscopic simulation of evacuation processes on passenger ships. Proceedings on the 4th International Conference on Cellular Automata for Research and Industry: Theoretical and Practical Issues on Cellular Automata. Oct 4-6, 2000; pp. 63-71, Germany.

[31] Quarantelli EL. Sociology of Panic. In: International encyclopedia of the social and behavioral sciences. Smelser NJ, Baltes PB, Eds. Elsevier Science: London 2001.

[32] Christakos CK. Simple pedestrian simulator using node-edge graphs for floorplan models. In: Proceedings of the Summer Simulation Multiconference. Jul 30-Aug 3, 2006; pp. 1-5, Calgary, Canada.

[33] Nagel K, Schreckenberg M. A cellular automaton model for freeway traffic. J Phys 1992; 2: 2221-9.

[34] Zhang J, Wang H, Li P. Cellular automata modeling of pedestrians crossing dynamics. J Zhejiang Univ Sci 2004; 5(7): 835-40.

[35] Antonini G, Venegas S, Thiran JP, Bierlaire MA. Discrete choice pedestrian behavior model for pedestrian detection in visual tracking systems. In: Proceedings of Advanced Concepts for Intelligent Vision Systems. Aug 31-Sep 03, 2004; pp. 1-8, Brussels, Belgium.

[36] Mauron L. Pedestrians simulation methods, Ph.D. Thesis, Swiss Federal Institute of Technology ETHZ, 2002.

[37] Hoogendoorn SP, Bovy PH, Daamen W. Microscopic pedestrian way finding and dynamics modeling. In: Schreckenberg M, Sharma S, Eds. Pedestrian and Evacuation Dynamics, Duisburg 2002; pp. 123-54.
[38] Yang Q, Koutsopoulos H. A microscopic traffic simulator for evaluation of dynamic traffic management systems, Transp Res 1997; 4 (3): 113-29.

[39] Cavens D, Gloor C, Illenberger J, Lange E, Nagel K, Schmid W. Distributed intelligence in pedestrian simulations. Pedestrian and Evacuation Dynamics; Springer 2007; pp. 201-12.

[40] Mulyana WM, Gunawan TS. Hajj crowd simulation based on intelligent agent. In: Proceedings of International Conference on Computer and Communication Engineering. May 11-12, 2010; pp. 1-4, Kuala Lumpur.

[41] Zadeh LA. Fuzzy sets. Inform Control 1965; 8 (3): 338-53.

[42] Zadeh LA. Discussion: Probability theory and fuzzy logic are complementary rather than competitive. Technometrics 1995; 37(3): 271-6. Retrieved from http://www.jstor.org/stable/1269908

[43] Wang, YM, Yanga JB, Xua DL, Chinc KS. On the centroids of fuzzy numbers. Fuzzy Sets Syst 2006; 157: 919-26.

[44] Alizadeh R. A dynamic cellular automaton model for evacuation process with obstacles. Saf Sci 2011; 49: 315-23.

[45] Weng WG, Chen T, Yuan HY, Fan WC. Cellular automaton simulation of pedestrian counter flow with different walk velocities. Phys Rev E Stat Nonlin Soft Matter Phys 2006; 74: 036102.

[46] Biham O, Middleton AA, Levine D. Self-organization and a dynamical transition in traffic-flow models. Phys Rev 1992; 46: R6124.

[47] Dorigo M, Maniezzo V, Colorni. Ant system: Optimization by a colony of cooperating agents. IEEE Trans Systems Man Cybernet 1996; 26(1):29-41.

[48] Gipps PG, Marksjo B. A micro-simulation model for pedestrian flows. Math Comput Simul 1985; 27: 95-105.

[49] Schreckenberg M, Wolf DE. Traffic and Granular Flow, World Scientific: Singapore 1998.

[50] Brilon W, Ponzlet MW. Application of traffic flow models. traffic and granular flow. In: Wolf DE, Schreckenberg M, Bachem A, Eds. Springer: London 1995.

[51] Hall FL, Montgomery FO. The investigation of an alternative interpretation of the speed-flow relationship for U.K. motorways. Traf Eng Control 1993; 34: 420-5.

[52] Koshi M, Iwasaki M, Ohkura I. Some findings and an overview on vehicular flow characteristics. In: Proceedings of 8th International Symposium on Transportation and Traffic Theory, (ISTTT'83). University of Toronto Press: Ontario 1983; p. 403.

(C) Boulmakoul and Mandar; Licensee Bentham Open.

This is an open access article licensed under the terms of the Creative Commons Attribution Non-Commercial License (http://creativecommons.org/licenses/by-nc/ 3.0/) which permits unrestricted, non-commercial use, distribution and reproduction in any medium, provided the work is properly cited. 